\title{
Legal hopes: enhancing resilience through the external cultivation of positive emotions
}

\author{
KATHY ABRAMS \\ Herma Hill Kay Distinguished Professor of Law at UC-Berkeley \\ School of Law
}

\author{
AND \\ HILA KEREN \\ Professor of Law at Southwestern Law School
}

\begin{abstract}
L egitimated by rationality and grounded in claims of dispassion and objectivity, the law has been reluctant to acknowledge its relationship to the emotions. ${ }^{1}$ When legal actors have recognized emotion, their focus has often been on negative emotions - from vengeance to shame or regret - and the way that their amplification, control, or redirection can shape doctrine, or contribute to the achievement of legal goals. ${ }^{2}$ Legal thinkers have been slower to embrace a role in which legal actors use law to achieve emotional goals, rather than using emotion to achieve legal goals. ${ }^{3}$ This has been true, a fortiori, where the goal of legal action is to foster positive emotions, such as hope, interest, joy, or feelings of affection or solidarity. The spectre of an intrusive state coercing the performance of sham emotions ${ }^{4}$ has slowed exploration in this potentially productive area. In this article, we challenge legal scholars to rethink this reluctance, by exploring one context in which the legal cultivation of positive emotions may prove particularly useful. This context is the fostering of resilience, defined either as the capacity for normal development in circumstances of chronic stress or deprivation (e.g. extreme poverty or parental violence or drug use), or as the ability of less systematically disadvantaged individuals to respond productively to more acute stressors (e.g. unemployment, or death or illness of a family member). A growing psychological literature has highlighted the role of positive emotions in producing resilience and has emphasized the role of habituation in fostering such emotions. Drawing on our earlier work on the legal cultivation of hope, we argue that habituation to positive affective responses can be achieved not only by self-regulation and therapeutic intervention, but by programmatic interventions prescribed by law.
\end{abstract}

Our paper is divided into three parts. In the first part we describe the major strands of psychological literature on resilience (and the related concept of coping), and we highlight the emerging view of the positive emotions as a critical resource for resilient adaptation to

1 S Bandes (ed), The Passions of Law (New York University Press 1999); K Abrams and H Keren, 'Who's Afraid of Law and the Emotions? (2010) 94 Minnesota Law Review 1997.

2 See, for instance, M C Nussbaum, “The Secret Sewers of Vice”: Disgust, Bodies and the Law' in Bandes (n 1) 19; D M Kahan, 'The Progressive Appropriation of Disgust' in Bandes (n 1) 63; S Garvey, 'Can Shaming Punishments Educate?' (1998) 65 University of Chicago Law Review 73; C Guthrie (2008). 'Carhart, Constitutional Rights and the Psychology of Regret' (2008) 81 Southern California Law Review 877.

3 Abrams and Keren (n 1).

4 C Sanger, 'The Role and Reality of Emotions in Law' (2001) 8 William and Mary Journal of Women and Law 107. 
adverse circumstances. In particular, we underscore some recent work which suggests that positive emotions may be fostered by a process of habituation.

In the second part, we describe our work on the positive emotion of hope. We argue that hope - which we define as the capacity to conceive and project oneself toward a distant but not impossible goal - can be cultivated through law by a sustained process of habituation. We describe a framework through which a legal actor or an institution designed by law can foster hope in individuals or groups, a framework which may have application to other positive emotions as well.

In the third part, we identify three themes in the potential relationship between our work on the legal cultivation of hope and the psychological literature on resilience. First, the psychological literature may affirm and offer empirical support for important dimensions of our framework. Second, our framework for the external cultivation of hope, through legal representation or institutional design, may extend current psychological approaches, which focus on individual self-regulation or intervention through the limited context of therapeutic relationships. Third, legal interventions aimed at providing individuals or groups with the habituation to hope may offer psychologists a context in which to explore habituation to positive emotions and its contribution to resilience in all its 'real world' complexity.

\section{Resilience: a brief tour of a burgeoning field}

Why do some individuals respond to adversity in a way that permits them to flourish, while others struggle or fail to adapt? This question promises to be central to the study of human vulnerability; it enables scholars to glimpse a trajectory out of systematic disadvantage for some who are subject to structural inequalities, violence, trauma, or natural disasters. It may also permit policy makers to design interventions that draw on this experience by formulating strategies that provide or mobilize the distinctive range of resources utilized by these more successful adapters. Given these stakes, it is not surprising that the study of resilience, in both children and adults, has been a lively focus of inquiry in psychology, as well as in allied fields such as social work and education. Notwithstanding these potential benefits, however, the analysis and cultivation of resilience have not been a focus of investigation in law.

\section{EARLY LITERATURE ON RESILIENCE}

Until recently, the psychological literature on resilience could be divided into two broad categories. One body of literature, which was more developmental in its emphasis, described resilience as the adaptive functioning of children or young adults who had been exposed to conditions of adversity. ${ }^{5}$ Adaptive functioning was most frequently described as normal development, understood in cognitive, psychological/affective, or behavioural terms. ${ }^{6}$ The conditions of adversity surmounted by resilient youth ranged from the physical or psychological illness or drug-dependence of a parent to systematic socio-economic disadvantage. ${ }^{7}$ This work focused on 'factor-specific' analyses (which used multivariate

5 S S Luthar, D Cicchetti and B Becker, 'The Construct of Resilience: A Critical Evaluation and Guidelines for Future Work' (2000) 71 Child Development 543; A S Masten, 'Ordinary Magic: Resilience Processes in Development' (2001) 56 American Psychologist 227.

6 B Egeland, E Carlson and L A Sroufe, 'Resilience as Process' (1993) 5 Development and Psychopathology 517. A similar framework is applied to adults in G A Bonanno, 'Loss, Trauma, and Human Resilience: Have We Underestimated the Human Capacity to Thrive after Extremely Aversive Events?' (2004) 59 American Psychologist 20.

7 Luthar et al (n 5); M Conrad and C Hammen, 'Protective and Resource Factors in High- and Low-risk Children: A Comparison of Children with Unipolar, Bipolar, Medically Ill, and Normal Mothers' (1993) 5 Development and Psychopathology 593. 
statistics to test for relations between risk, development or competence achieved, and specific personal or environmental factors that may protect individuals from potentially negative influences on development), or on 'person-specific' analyses (which compared people with different outcome profiles along specific criteria to determine what naturally occurring clusters of factors tended to differentiate resilient children). ${ }^{8}$ These studies, in the main, described resilience as a product of both individual attributes, such as 'cognitive and self-regulation skills' or 'positive views of self' and of group-based or relational features of the surrounding environment, such as 'connections to competent and caring adults in the family and community'. 'This literature, which depicted its subjects as situated individuals subject to group-based influences and interventions, was often cryptic or elliptical in its treatment of the emotions. While works within this literature sometimes described the adaptive functioning of its young subjects along an affective axis, this focus was frequently limited to observing the presence or absence of clinical symptoms, such as anxiety or depression. ${ }^{10}$ And while particular scholars sometimes identified affective influences, such as 'emotionally responsive [parental] caregiving', ${ }^{11}$ or 'connections to . . caring adults', 12 most did so without analysing at depth the emotional dimensions of these connections. ${ }^{13}$ Although resilience in this literature was often described as a process, ${ }^{14}$ this was a developmental process, which did not entail discussion of patterns of habituation.

A second body of literature analysed coping in relation to stress. This literature focused on adults who experienced either long-term stressors, such as the illness or death of a spouse, ${ }^{15}$ or short-term stressors, such as work or financial pressures, or any set of developments that temporarily taxed an individual's 'resources', broadly defined. ${ }^{16}$ This literature was more explicit and analytical in its approach to the emotions. This focus is exemplified by the work of Richard Lazarus, who analysed coping through a 'cognitive theory of the emotions'. ${ }^{17}$ In this account, the ability of a particular person to cope with adversity is conditioned by his or her 'cognitive appraisal' of her situation: 'through cognitive appraisal the person evaluates the significance of what is happening for his or her

8 Masten (n 5).

9 Ibid.

10 R D Felner et al, 'Socioeconomic Disadvantage, Proximal Environmental Experiences, and Socioemotional and Academic Adjustment in Early Adolescence: Investigation of a Mediated Effects Framework' (1995) 66 Child Development 774.

11 Egeland et al (n 6).

12 Masten (n 5).

13 Some work is more ambivalent with respect to its exploration of the emotions. Egeland et al (n 6) associate 'emotionally responsive parenting' with performance on a particular experimental measure administered to a parent and child when the child is 42 months old. However, the article also uses the work of John Bowlby (Attacbment (Penguin 1984)) to try to explain the contribution of this attribute. Citing Bowlby, Egeland notes that 'emotionally responsive parenting is thought to assist the child with regulating emotional response and developing confidence in the supportive presence of others'. In this account, the child interacts with the caregiver over time and comes to feel that he is 'lovable and worthwhile'. Although Bowlby's work is on attachment rather than resilience, and Egeland at al's attention to it reflects an emotional focus which seems atypically sustained for this body of literature, this notion of developing confidence and capacity for selfregulation over time, through the supportive response of a caregiver, may preview later works: see, for instance, M M Tugade, 'Positive Emotions and Coping: Examining Dual-process Frameworks of Resilience' in S Folkman (ed), The Oxford Handbook of Stress, Health and Coping (OUP 2011) 186; J T Moskowitz, 'Coping Interventions and the Regulation of Positive Affect' in ibid 407.

14 Egeland et al (n 6)

15 Bonanno (n 6).

16 R S Lazarus and S Folkman, Stress, Appraisal, and Coping (Springer 1984).

17 Ibid. 
wellbeing. ${ }^{18}$ This process may encompass a 'preliminary' appraisal of whether the situation or event is positive, irrelevant, or stressful to the individual and, if the event is stressful, a 'secondary' appraisal of 'what might and can be done'. ${ }^{19}$ Both appraisals, in Lazarus's account, rest on an individual's commitments, or judgments, about what is important, and beliefs about the world and his or her own capacities. These appraisals are also intertwined with emotions. Emotions - such as the feeling of fear, anger, or excitement that may emerge prior to purposeful cognitive evaluation - may provide the first signal that an individual is facing a circumstance that requires assessment. Emotions may also emerge as the products of cognitive appraisal. A person who believes that he or she has the ability to resolve a challenge consistently with his or her goals may feel pride, confidence, or relief. One who believes that the circumstances are beyond his or her ability to cope may feel fear or panic. One who believes that he or she cannot respond effectively to the surrounding circumstances without violating one or more of his or her existential commitments may feel frustration or some form of emotional ambivalence. Emotions may help processes of coping (hope or confidence or indignation may fuel perseverance in facing a difficult situation), or hinder them (outrage or fear or excitement may make it difficult to conceive or execute a plan). They may also trigger further cognitive reassessment in an ongoing process. For Lazarus, improved coping can arise from helping the individual to engage in cognitive reappraisal - that is, to think differently about his or her capabilities or goals, so that there is less anxiety experienced, or more confidence in the ability to respond to the circumstances eliciting it. Coping can also arise from helping the individual to regulate the effects of the emotion, through such strategies as distraction or distancing. ${ }^{20}$ Although Lazarus and Folkman state that there is no empirical basis at present for preferring one of these approaches to the other, the weight of their analysis is applied to the strategy of cognitive reappraisal. ${ }^{21}$

While this theory reflects nuance in its cognitive theory of the emotions generally, it does not take a position on the value or potential contribution of any particular set of emotions. Emotions are analysed as accurate or inaccurate reflections of the circumstances, or the cognitive assessments, that produced them, or as assisting or hindering efforts to cope with particular problems. The answer to situationally problematic emotions lies in helping the individual to engage, first, in cognitive reappraisal and, perhaps secondarily, in the down-regulation of situationally inappropriate or unhelpful emotions. This help is provided primarily within the context of a therapeutic relationship. As distinct from the literature on child and adolescent resilience, this cognitively based view of coping tends to focus on the individual and his or her response to stressful circumstances rather than the institutions or relationship in which he or she is embedded; it seeks to alter cognitive appraisals through a therapeutic relationship (and ultimately, through self-regulation) rather than by addressing the individual's surrounding environment.

\section{THE ROLE OF POSITIVE EMOTIONS IN BUILDING RESILIENCE: NEW DEVELOPMENTS}

Over the two last decades, however, theorists have shown a new awareness of the special role played by the positive emotions in explaining patterns of resilience. Their work was inspired by Barbara Fredrickson's 'broaden-and-build' theory, which offers a general

\footnotetext{
18 Lazarus and Folkman (n 16).

19 Ibid 35 .

20 Ibid 319.

21 Ibid 374.
} 
understanding of the unique importance of the positive emotions. ${ }^{22}$ The broadening influence of the positive emotions allows people to think or act in ways that were not previously available to them. For example, a person who is experiencing joy or gratitude may consider engaging in new or unfamiliar activities, such as seeking the company of others, or engaging in a creative project. ${ }^{23}$ The building effect follows when the exploration of such expanded possibilities results in a growth of new personal resources. A social interaction might lead to establishing friendships, or an artistic project could develop into a hobby or a field of interest. Although Fredrickson's theory was not originally developed in the context of resilience, the broaden-and-build model has the potential for explaining individual differences in coping with negative events and prolonged hardships; it therefore offers an important dimension to the analysis of resilience.

One way the broadening effect of the positive emotions contributes to resilient trajectories is by interrupting and tempering the negative emotions that inhere in adversity and exacerbate it. The presence of positive emotions in conjunction with negative emotions has the power to 'undo' some of the negative emotions and thereby enable better and easier adaptation. For example, one study of coping with bereavement documented better adjustment by those individuals who, in the course of grieving, experienced and expressed some positive emotions, as demonstrated by smiling and laughing. ${ }^{24}$ In other words, having access to some positive emotions, even if they are only briefly and moderately experienced, can assist in handling times of crisis by down-regulating the negative emotions produced by the crisis.

The broadening effect of the positive emotions also expands access to cognitive and behavioural pathways that tend to be blocked or limited by the 'tunnel vision' that typifies the distressed. If effective coping requires cognitive reappraisal of the meaning of a problem, for example, as many scholars have suggested, ${ }^{25}$ then positive emotions can facilitate such cognitive effort. By offering a break from the negative orientation that tends to be produced by a personal crisis or a more sustained form of distress, positive emotions can make the affected individual more available for, and capable of engaging in, the longterm thinking and problem-solving that are essential to resilient coping.

The building effect of the positive emotions is also a vital component of the resilient response. Positive emotions have the potential to create and enhance resources that are requisite to successful coping. Supportive relationships and higher levels of self-esteem, for example, have consistently been correlated with resilience. ${ }^{26}$ Recurrent positive emotions play a major role in developing and restoring these interpersonal resources; they also contribute to the building and sustaining of physical resources, such as health and energy, and mental resources, such as motivation and attentional focus. ${ }^{27}$

These valuable contributions of the positive emotions to resilience suggest the need for interventions that can increase positive affect. Until very recently, the small group of scholars interested in the effects of positive emotions on coping focused on deliberate, selfadministered efforts to elicit positive emotions. These strategies included keeping gratitude

22 B L Frederickson, 'What Good are Positive Emotions?' (1998) 2 Review of General Psychology. Special Issue: New Directions in Research on Emotion 300.

23 B L Fredrickson and C Branigan, 'Positive Emotions Broaden the Scope of Attention and Thought-action Repertoires' (2005) 19 Cognition and Emotion 313.

24 D Keltner and G A Bonanno, 'A Study of Laughter and Dissociation: Distinct Correlates of Laughter and Smiling during Bereavement’ (1997) 73 Journal of Personality and Social Psychology 687.

25 Lazarus and Folkman (n 16).

26 Masten (n 5).

27 Moskowitz (n 13). 
journals, meditating, exercising and trying to find positive meaning in negative events. These individual strategies situate interventions primarily within a therapeutic paradigm. They operate on individuals and they produce change by addressing the cognitive or behavioural patterns of individuals, rather than addressing their surrounding environment.

A recent work by Michele Tugade adds a new and promising path to the cultivation of positive emotions. ${ }^{28}$ Arguing that generating positive emotions does not necessarily require conscious efforts, Tugade illuminates the possibility of 'automatic activation' of such emotions. He begins by observing that certain 'bottom-up' stimuli automatically produce positive emotions: a cup of hot tea may fuel contentment; or a random smile from a passerby may engender a sense of social connectedness. He then argues that such automatic responses can be triggered not only by random interactions, but by habituation: from repeated, deliberate efforts to generate positive emotions. Practising loving kindness meditation, which intentionally evokes positive emotions, can produce a state in which positive emotions emerge naturally, without purposive effort. ${ }^{29}$ Purposefully distracting oneself from negative emotions over years and decades, as work cited by Tugade demonstrates, may trigger among older adults an automatic shift of attention from negative stimuli to positive stimuli. ${ }^{30}$ Another recent work by Judith Moskowitz supports Tugade's new emphasis on the power of deliberate efforts to produce automatic activations of positive emotions. As Moskowitz argues, 'for an intervention to be effective, the individual needs to make the targeted behaviors a habit. ${ }^{31}$ For example, interventions that led individuals to integrate meditation, volunteering work, art classes or social meetings into their routine have been shown to increase positive affect. Importantly, although many interventions may yield temporary positive emotions, only continuing the patterns described above can defeat individuals' inclination to adapt back to their set point.

These recent works of Tugade and Moskowitz remain within the individual or therapeutic frame: they support cognitively based self-regulation by individuals, but do not aim to cultivate positive emotions in others by intervening in their surrounding environments. But they offer a new direction that, in our view, reflects promise, namely the enhancement of coping or resilience by purposeful efforts to cultivate positive emotions through habituation. In the following section, we consider a different means of cultivating habituation to positive emotion, namely by external regulation of the individual's environment through the vehicle of law. In this context we discuss work that we have done on the cultivation of hope in individuals and groups suffering systematic disadvantage. In this account, law serves to structure environments which engender 'habits of hope': these habits, over time, foster in individuals or groups an independent ability to activate hopeful emotions.

\section{Cultivating hope through law}

In our 2007 article, 'Law in the Cultivation of Hope', we examined the role of law in supporting the emergence of one positive emotion, hope, in groups facing adversity, particularly race-based stigma and socio-economic disadvantage. ${ }^{32}$ We described hope as the capacity to 'aspir[e] to a goal that is arduous and difficult but possible' and work toward the means of its achievement. The complex combination of the affective and the cognitive that

28 See n 13.

29 B L Frederickson et al, 'Open Hearts Build Lives: Positive Emotions, Induced through Loving-kindness Meditation, Build Consequential Personal Resources’ (2008) 95 Journal of Personality and Social Psychology 1045.

30 Tugade (n 13).

31 See n 13.

32 K Abrams and H Keren, 'Law in the Cultivation of Hope' (2007) 95 California Law Review 319. 
is involved in hope includes the courage and imagination necessary to grasp a future prospect that departs from one's present circumstances, the means-ends rationality necessary to formulate means to this end, the persistence to move forward in the face of failures, missteps and opposition, and the willingness and resourcefulness to draw on or mobilize the support of others. As one hopes - or pursues particular hopes over time - one develops a disposition of hopefulness, which not only fuels particular actions but conditions the ways that one looks at difficulties or challenges. Drawing on the insight of pragmatist philosopher Patrick Shade that hope and hopefulness can emerge or can be supported by a process of habituation, ${ }^{33}$ we considered the possibility that individuals or institutions can engage in the 'cultivation of hope' - that is, can engender hope in those who lack it. Highlighting examples both inside and outside of law, we described a process by which one individual, already possessed of a hopeful disposition, can cultivate hope in others.

In this context, we proposed a framework for this process of cultivation which proceeded in several steps. First, the cultivator must 'communicat[e] recognition and vision'. Recognition means signalling that he or she sees the prospective hoper as a full human subject, with multifaceted capacity and individual personality. Vision means helping the prospective hoper to see that aspects of his or her situation could be different. Second, the cultivator must 'introduc[e] an activity that allows for individuation'. This step helps the prospective hoper to recognize his or her own capacity or talent, and to begin to express it in concrete ways that the hoper and others can see. In the examples we develop, this 'activity' could be as varied as taking notes in a group meeting, depicting one's immediate environment through photography, or telling one's story of legal injury to the media. As the hoper's activity begins to unfold, the cultivator must also 'provide resources' where necessary to help the hoper develop a sense of possibility or efficacy in relation to his or her endeavour: this might mean providing material resources, or access to networks or other communities with similar interests. The cultivator must also 'support [the] agency' of the prospective hoper. Those who lack hope often believe that they are not capable of directing their own course, or of efficacy in implementing even short-term goals. In this phase, the cultivator fosters a sense of greater capability and control by setting tasks or offering opportunities that demand progressively more self-assertion or self-direction, or supports the new hoper in meeting comparable challenges when they are presented by the surrounding environment. Finally, the cultivator must 'foster [a sense of] solidarity' with the new hoper, and among all the other hopers with whom the cultivator may be involved (if the cultivation effort is proceeding with a group larger than one). A sense of isolation is central to the despair, enervation and paralysis that are the opposite of hope. A feeling of solidaristic connection with others counters that sense of isolation, as well as providing the hoper with mentors and fellow-travellers: it may also permit the hoper to re-activate earlier phases of the cultivation process (reliance on resources or supports for agency, for example) when he or she encounters obstacles or disappointments.

Although we initially describe the cultivation of hope as an individual effort, we subsequently argue that the same framework can be particularly well utilized by the law. This is sometimes because lawyer-client relationships provide the occasion for an individual cultivation of hope. For instance, Julie Su, an attorney with the Asian Pacific American Law Center of Southern California, helped to cultivate hope among the workers whom she represented in an action against the distributors of items produced by sweatshop labour. Su framed the action as one dimension of a community-organizing campaign, in which she supported workers in telling their own stories to the media, enlisted them in particular aspects of the litigation effort and fostered connections among Thai and Mexican workers

33 P Shade, Habits of Hope: A Pragmatic Theory (Vanderbilt University Press 2001). 
who laboured under different conditions and spoke different languages. Lawyers who represent clients on death row, as we have discovered in another research project, ${ }^{34}$ may also engage in this kind of individual cultivation, supporting the hopes of their clients not simply for parole or a new trial, but for a meaningful life that can be lived in prison - by supporting their interests in continuing education, in hobbies or artistic efforts, or in mentoring other prisoners, and by helping them to achieve as much agency as possible in relation to prison conditions and contact with friends and family. More often, however, the law becomes a potent instrument in the cultivation of hope because it is capable of structuring institutional environments that can support a habituation to hope and hopefulness.

In our 2007 article, we explored the example of Project Head Start, a nationwide preschool programme that was initiated by law under the Johnson Administration. Head Start not only offered an educational foundation to children living in circumstances of systematic deprivation, but also fostered the hope of their mothers, as it involved them in programmes of local Head Start centres. The requirement that the mothers volunteer to take part in the administrative work of the centres and assist with classes gave them a set of responsibilities that permitted them to draw on whatever strengths they possessed, and over time habituated them to feelings of capability, contribution and greater control. The structure of mentorship that the centres provided through ongoing contact with supervising teachers and other mothers offered newer mothers supports for their growing agency; they could rely on information, advice and reassurance as they took on increasingly demanding roles. Mothers could also draw on resources: the material resources of the school, the social networks provided by other parents and teachers and emotional resources for addressing challenges both inside and outside of the school environment. Finally, gradual immersion in the educational and administrative work of the centre, buttressed by supportive mentoring from teachers and other mothers, created both vertical and horizontal bonds of solidarity, trust and affection among members of the Head Start community.

\section{Legal hopes and the psychological literature of resilience}

The framework for cultivating hope that we advanced is in some ways a hypothesis - based on a series of case studies that we investigated at length - about how interventions structured by law might be used to foster the positive emotion of hope. ${ }^{35}$ Our review of the emerging literature of resilience suggests important relationships between that literature and our thinking about the cultivation of hope.

Although our understanding of how we might cultivate hope through law was drawn from distinct philosophical, legal and sociological literatures, we see it as reflecting many of the insights embodied in the psychological literatures on resilience. With the literature on coping with stress, and on the value of the positive emotions, we share the insight that emotions (whether understood as the products of cognitive appraisal or as emerging in more automatic ways) are critical to the way that one responds to adversity. Like these literatures, our work suggests that positive emotions might have particular value: first, in broadening perceptions of one's circumstances and possible responses; and second, in helping one to develop and sustain resources crucial to coping, such as social support and a sense of self-efficacy. Yet, like the developmental literature on the determinants of resilience, our work recognizes that the individualized or therapeutic emphasis of these

34 Work currently in progress.

35 Our perhaps atypically broad understanding of hope means that it encompasses other affective responses described as positive emotions in the psychological literature: interest, joy, love. So the application of something like the steps we describe could potentially result in the cultivation of a number of positive emotions (as they are characterised in the literature), and not simply hope. 
more emotionally focused literatures on coping needs to be supplemented with an emphasis on the environmental factors that can condition adversity and enable a response. Our analysis shares with a sociological literature on coping the recognition that many forms of adversity are neither random nor wholly individuated but rather the product of structural factors, such as systemic forms of inequality. ${ }^{36}$ It also acknowledges, with the developmental literature on resilience, the insight that how individuals respond to adversity is not simply a function of internal capacities (including cognitive response), but also of their connection with various resources in the surrounding environment. ${ }^{37}$ This last insight makes the turn to law, which is capable of structuring or regulating relations between individuals and their environments, a plausible and necessary move. Finally, with the emerging literature on positive emotions, our work shares a modus operandi: the notion that habituation may be a key in supporting the emergence of positive emotions. Yet, it moves that emphasis on fostering habituation beyond the therapeutic environment, into settings where individuals and groups work, learn and dispute the conditions under which they live.

In this section, we offer three themes that characterize those relationships. First we argue that the literature on resilience offers support for our hypothesis, including our focus on habituation and several dimensions of our framework of cultivation. Second, we suggest that our work adds a dimension that is currently not developed in the resilience literature, in that it highlights the use of external intervention to foster positive emotions, either by the individual or through the design of institutions. Third, we suggest that our focus on the cultivation of positive emotions through law - particularly those contexts in which law structures programmatic interventions - may provide social scientists with an opportunity to investigate the generation and the effects of positive emotions in practical settings aimed at responding to circumstances of adversity. Each of these relationships suggests that law may be of value in cultivating the positive emotions that may support the development of resilience.

\section{SUPPORT FOR THE FRAMEWORK OF EXTERNAL CULTIVATION}

Given these common premises, the literatures on resilience elaborate on, and offer empirical support for, a number of the hypotheses we have drawn from our particular cases or examples. First, the recent research on positive emotions and habituation reinforces a central theme in our work, namely the need to foster habits of hope. The cultivation of hope operates through a sustained process, that is as much about fostering confidence and a sense of possibility through repetition and practice as it is about inciting purposeful cognitive readjustments.

Second, the literatures on resilience also support specific dimensions of our framework of cultivation. For example, these literatures underscore the importance of providing to prospective hopers an activity allowing for individuation. This is in some ways the most surprising dimension of our theory: how does hope arise from learning to use a camera, or helping with litigation documents, or assisting in a preschool class? The resilience literature points to several dynamics that support this connection. One is the centrality of engagement. Hobfoll notes, for example, that a sense of engagement is critical to the experience of many positive emotions, such as excitement and fulfilment. ${ }^{38}$ Finding an activity in which prospective hopers can take part is a way of bringing forward their capacities for attention, enjoyment and commitment - in short, engaging them. The focus

36 L I Pearlin, 'The Sociological Study of Stress' (1989) 30 Journal of Health and Social Behavior 241.

37 Masten (n 5); S E Hobfoll, 'Conservation of Resources Theory: Its Implication for Stress, Health, and Resilience' in Folkman (n 13) 127.

38 Hobfoll (n 37). 
on an activity allowing individuation also permits them to deploy what they perceive as their strengths, another strategy that is associated in the resilience literature with the emergence of positive emotions. ${ }^{39}$ One Head Start mother illustrated this effect in describing her decision to run for secretary of her local centre's policy council: 'You see, I'm good with paperwork. I'm good. And I got my notebooks together. And I got the plastic covers ... and I had categories set up, and I had it just perfect . . . I conducted my little position like a professional.' Finally, sustained focus on a specific activity can be a vehicle for helping people to see their prospects differently, through the process of habituation. This dynamic is consistent with Tugade's 'dual processing' theory of positive emotions - that they are capable of emerging both deliberately and automatically. ${ }^{40}$ But our work suggests that the vector may move in both directions. Not only do repeated efforts at reappraisal lead to a more habitual affective response; but the habitual performance of a particular activity or task may prompt affective responses that then lead to a gradual cognitive reappraisal. As another Head Start mother observed:

It's built my self-esteem up . . . I feel better about myself . . . Like going to Governing Board meeting and sitting beside the superintendent . . . Then you feel, well you are worth something. And I didn't used to feel like that.

The psychological literature on resilience also confirms a second element of our framework, namely the importance of providing resources in fostering hope. Hope may not intuitively seem to be connected with resources, but when it is defined as the ability to conceive and project oneself toward a distant and difficult goal, the value of resources becomes more apparent. Both the developmental literature on resilience and the work on adult coping highlight the centrality to successful adaptation of various forms of resources. These may be material resources, or experience with navigating institutions, or social network-based support. ${ }^{41}$

Yet another theme in the resilience literature correlates with the element of 'solidarity' in our framework. Forms of interpersonal connection, such as responsive parenting and effective mentorship, are critical in the developmental literature on resilience; 42 connection with others is both required for and enhanced by the broaden-and-build framework of positive emotions. ${ }^{43}$ Solidarity is a key to cultivation in all of our examples, whether the connections among Head Start mothers, and their relations with their teacher-mentors, or the relationship between Julie Su and the immigrant workers, or the relation of Thai workers to their Mexican counterparts. Solidarity defeats the main enemy of hope isolation: it permits these prospective hopers to grow in competency and find emotional support to sustain them in the face of obstacles.

Finally, these literatures affirm the support for agency which is a central dimension of our framework. Cultivators from Zana Briski, who brought cameras to the children of the red-light district in Calcutta, to the teacher-mentors of Head Start support new hopers as they expand the scope of their activity and independence, gaining a sense of efficacy and self-direction. The contribution of self-efficacy to resilience in the face of adversity is a

39 M E P Seligman et al, 'Positive Education: Positive Psychology and Classroom Interventions' (2009) 35 Oxford Review of Education 293; K J Reivich, M E P Seligman and S McBride, 'Master Resilience Training in the US Army' (2011) 66 American Psychologist 25.

40 Tugade (n 13).

41 Masten (n 5); Luthar et al (n 5); Conrad and Hammen (n 7); Hobfoll (n 37).

42 Luthar et al (n 5); Masten (n 5); Egeland et al (n 6).

43 Frederickson (n 22); Frederickson and Branigan (n 23). 
point made across the various psychological literatures. ${ }^{44}$ It is correlated with greater resilience among children who experience disadvantage, ${ }^{45}$ contributes to adult capacity to cope with trauma ${ }^{46}$ and is regarded in some work as a critical resource in coping. ${ }^{47}$ Our work reflects the additional 'dual process' insight that agency may be fostered both through cognitive reappraisal and through habituation. ${ }^{48}$ Julie Su helped workers to understand that their own voices - which the workers had devalued for their lack of English fluency and legal expertise - in fact offered the most compelling account of the abuses they had endured. ${ }^{49}$ This cognitive reappraisal fuelled a greater sense of efficacy as well as feelings of pride, confidence and hope. In the main, however, workers' agency grew through habituation: the workers developed a sense of competency and control over their lives, as they helped, day after day, in the production of litigation documents, or spoke, again and again, to members of the press.

\section{THE EXTERNAL CULTIVATION OF POSITIVE EMOTIONS}

As we have seen, psychologists have recently argued that the value of positive emotions in building resilience justifies deliberate efforts to foster such emotions in times of distress. However, these calls for intervention have focused on efforts by individuals to up-regulate their own positive affect: independently, through practices such as meditation or gratitude diaries, or with the guidance of a therapist. ${ }^{50}$ Our own work builds on this effort, by moving the emphasis on deliberate cultivation beyond the individual self-regulation or the therapeutic relationship. We describe and theorize the ability of individuals and institutions to cultivate positive emotions in others, using law as a vehicle. By examining the work of lawyers, such as Julie Su, and legislated programmes, such as Head Start, we have come to believe in the feasibility of external contributions that utilise the robust personal and social resources of some to develop better coping resources in others who lack them. Our work suggests that society cannot rely solely on the resources available to individuals - particularly those who face circumstances of adversity - but rather should extend to its more vulnerable members a purposeful, active effort to enrich and strengthen their affective resources.

The framework we suggest can be perceived as extending recent work done by Hobfoll on resources and resilience. ${ }^{51}$ Highlighting the importance of environmental factors, such as socio-economic status, race and education, Hobfoll argues that disenfranchised individuals and families are limited in their ability to develop the resources that are essential to resiliency. Stating that 'for families who lack resources, the question of whether the future is bright or threatening takes a completely different meaning', Hobfoll suggests that some 'sociocultural ecologies' are so poor and demanding that they cannot provide any resources or support to their members and may in fact tax or erode the resources available to members to cope with specific challenges. Hobfoll goes so far as to observe that, under conditions of extreme deprivation, 'it is the greater social unit that must provide support':

44 Masten (n 5); S Folkman and J T Moskowitz, 'Positive Affect and the Other Side of Coping' (2000) 55 American Psychologist 647.

45 Masten (n 5); Luthar et al (n 5).

46 Folkman and Moskowitz (n 44).

47 Hobfoll (n 37).

48 Tugade (n 13).

49 J A Su, 'Making the Invisible Visible: The Garment Industry's Dirty Laundry' (1998) 1 Journal of Gender, Race, and Justice 405.

50 N L Sin and S Lyubomirsky, 'Enhancing Well-being and Alleviating Depressive Symptoms with Positive Psychology Interventions: A Practice-friendly Meta-analysis’ (2009) 65 Journal of Clinical Psychology: in Session 467.

51 Hobfoll (n 37). 
he references a legal intervention by the state of Mississippi in response to Hurricane Katrina, which produced a positive effect on mental health by regulating insurance payments. Yet, even Hobfoll stops short of explaining how greater social units can become supportive environments that have the capacity to enrich resources and expand them beyond the limited capacity of vulnerable individuals. A framework of external cultivation of hope through law can be seen as extending this recent idea: it explains the dynamics and specifies the elements that are likely to characterize effective external interventions. For example, by observing and theorizing the ability of the most reflective and innovative legal practitioners to cultivate hope in their clients by providing them with vision, activity, means and solidarity, our framework may assist those who are planning or designing external, legal interventions and point to new directions in the training of lawyers.

\section{Practical SetTings for InVestigating the generation AND EFFECTS OF POSITIVE EMOTIONS}

If the literatures on resilience offer explanation and empirical support for many of the patterns we identified in the cultivation of hope, the use of law in fostering positive emotions may offer a resource to social scientists as well. Studying environments structured by law that facilitate habituation to positive emotions may offer psychologists a means of testing and elaborating their claims about positive emotions. Thus, as Folkman and Moscowitz have observed:

[1] aboratory studies have provided provocative suggestions regarding the ways positive emotions may help people endure stress. But because constraints of the laboratory limit researchers' ability to simulate the meaning or duration of serious real-life stressors, we strongly encourage pursuing research under real-life circumstances, with all their complexity. ${ }^{52}$

Thus far, most research has focused on individuals suffering trauma or loss, such as the recently bereaved, ${ }^{53}$ caregivers for people with AIDS, ${ }^{54}$ or New Yorkers following 9/11. 55 Because these individuals are not part of any purposeful effort to foster positive emotions, their differences with respect to positive emotions and resilience are more likely to be the result of random stimuli, deliberate cognitive efforts, or serendipitous forms of habituation. Other studies have analysed the effects of 'positive psychology interventions' - that is, interventions aimed at fostering positive emotions through habituation - in the context of controlled therapeutic relations. ${ }^{56}$ Examining programmes structured by law to foster positive emotions, such as hope, might provide psychologists with an opportunity to study the effects of habituation 'under real life circumstances, with all their complexity'. Because legal theorists and actors are only beginning to discover this strategy, psychologists may need to begin with programmes such as Head Start, whose cultivation of hope in mothers was in some respects serendipitous, or at least secondary to its primary goal of educating preschool children. But as legal actors begin to experiment more purposefully with this framework, there may be new opportunities for psychologists to examine the processes by which lawyers and legally structured institutions help to foster positive emotions among groups who are their clients and beneficiaries.

52 Folkman and Moscowitz (n 44).

53 Tugade (n 13).

54 Folkman and Moscowitz (n 44).

55 B L Fredrickson et al, 'What Good are Positive Emotions in Crises? A Prospective Study of Resilience and Emotions Following the Terrorist Attacks on the United States on September 11th, 2001' (2003) 84 Journal of Personality and Social Psychology 365.

56 Sin and Lyubomirsky (n 50). 
In closing, we want to underscore our own hopefulness about the potential of law to enhance resilience by cultivating positive emotions. Two features of law work in concert to confer this potential. The first is that 'law' is a powerful social institution that may impact individuals' lives in numerous ways. Legislation, judicial decisions, statutorily-enacted programmes, legal representation, organisations established under law, legal incentives, legal declarations, special tribunals, legal instruments (such as contracts and trusts) and many more legally based tools together create the 'legal practice' in the broadest and richest sense of the term. The second promising feature of law, as broadly defined, is that it can be purposefully deployed to play this role in cultivating positive emotions and resilience, especially in distressed communities. Examples beyond those discussed here, drawn from our previous work, ${ }^{57}$ include: prison re-entry programmes, such as the Ready4Work Program; community lawyering efforts; international tribunals and truth commissions; alternative legal processes; and cohabitation agreements of same sex couples who are still prevented from marrying each other. Overall, we believe, in times of trouble, when resilience is essential, the law is too strong a tool to be left behind. 
